\title{
Restricted Combinatory Unification
}

DOI:

10.1007/978-3-030-29436-6_5

\section{Document Version}

Accepted author manuscript

Link to publication record in Manchester Research Explorer

\section{Citation for published version (APA):}

Bhayat, A., \& Reger, G. (2019). Restricted Combinatory Unification. In Automated Deduction - CADE 27 (Lecture Notes in Computer Science; Vol. 11716). https://doi.org/10.1007/978-3-030-29436-6_5

\section{Published in:}

Automated Deduction - CADE 27

\section{Citing this paper}

Please note that where the full-text provided on Manchester Research Explorer is the Author Accepted Manuscript or Proof version this may differ from the final Published version. If citing, it is advised that you check and use the publisher's definitive version.

\section{General rights}

Copyright and moral rights for the publications made accessible in the Research Explorer are retained by the authors and/or other copyright owners and it is a condition of accessing publications that users recognise and abide by the legal requirements associated with these rights.

\section{Takedown policy}

If you believe that this document breaches copyright please refer to the University of Manchester's Takedown Procedures [http://man.ac.uk/04Y6Bo] or contact uml.scholarlycommunications@manchester.ac.uk providing relevant details, so we can investigate your claim.

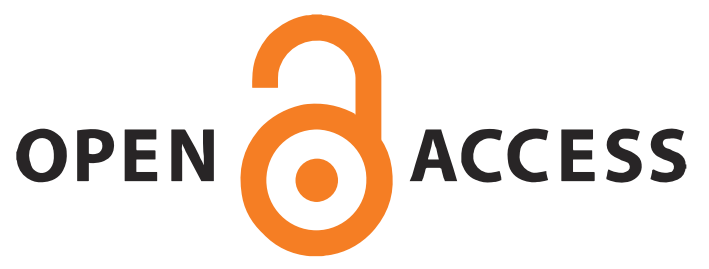




\title{
Restricted Combinatory Unification
}

\author{
Ahmed Bhayat and Giles Reger \\ University of Manchester, Manchester, UK
}

\begin{abstract}
First-order theorem provers are commonly utilised as backends to proof assistants. In order to improve efficiency, it is desirable that such provers can carry out some higher-order reasoning. In his 1991 paper, Dougherty proposed a combinatory unification algorithm for higher-order logic. The algorithm removes the need to deal with $\lambda$-binders and $\alpha$-renaming, making it attractive to implement in first-order provers. However, since publication it has garnered little interest due to its poor characteristics. It fails to terminate on many trivial instances and requires polymorphism. We present a restricted version of Dougherty's algorithm that is incomplete, terminating and does not require polymorphism. Further, we describe its implementation in the Vampire theorem prover, including a novel use of a substitution tree as a filtering index for higher-order unification. Finally, we analyse the performance of the algorithm on two benchmark sets and show that it is a significant step forward.
\end{abstract}

\section{Introduction}

Higher-order logic has many applications from the formalisation of mathematics through to uses in verifying the safety and security of computer systems. This has led to a growing interest in the automation of reasoning in higher-order logic. A successful step in this direction has been via translation to first-order logic and utilisation of first-order theorem provers, made possible by the high level of maturity and sophistication of such provers. Proof assistants such as Isabelle [23] and Coq [9] along with automated provers such as Leo-III [29], interact with first-order provers by translating their native logic into first-order logic [21]. These translations tend to be incomplete and suffer from a number of problems of which two of the most important are highlighted below. This paper addresses these problems with a higher-order unification algorithm for combinatory logic and its pragmatic realisation within the first-order Vampire theorem prover [17].

The translation of nameless or $\lambda$-functions is often carried out using combinators. However, when translating to monomorphic first-order logic, supported by most firstorder provers, an infinite set of combinators is required to guarantee completeness. Thus, most translation schemes suffice with including the combinators necessary to translate the $\lambda$-functions present in the input. Consider the somewhat contrived conjecture $\exists X: X b a=a$. On negation, this becomes $X b a \neq a$. As there are no ' $\lambda$ 's there would be no combinators present in the translation. Accordingly, the prover would be unable to synthesise the combinatory equivalent (which is $\mathbf{C K}$ ) of the $\lambda$-term $\lambda x y . y$ and would be unable to find a proof. Now consider the same conjecture, but assume that some combinator axioms are present in the first-order translation (this could be via the heuristic addition of combinator axioms, an option in Vampire). In this case, 
the axioms can superpose amongst themselves. For example, the $\mathbf{C}$ combinator axiom C $X Y Z=X Z Y$ could superpose onto the right hand side of the $\mathbf{S}$ combinator axiom $\mathbf{S} X^{\prime} Y^{\prime} Z^{\prime}=X^{\prime} Z^{\prime}\left(Y^{\prime} Z^{\prime}\right)$ to produce the equation $\mathbf{S}(\mathbf{C} X) Y^{\prime} Z^{\prime}=X\left(Y^{\prime} Z^{\prime}\right) Z^{\prime}$. A consequence of the combinator axioms has been derived that is of no use in proving the goal.

Both of these problems stem from attempting to achieve what the goal-oriented procedure of higher-order unification (HOU) does using the non-goal-oriented superposition calculus. Thus, there is a strong argument that introducing some form of higherorder unification into first-order provers would significantly improve their performance on problems generated by proof-assistants. This is particularly so if this can be achieved without harming performance on the first-order portion of the problems. We are not attempting to solve problems which require complex higher-order unifiers. Rather, the aim is to introduce limited HOU into a first-order prover to allow it to deal with 'nearly first-order' problems.

Consider for example, the TPTP problem NUM020^1. The problems posits the existence of the Church numeral 2 and requires provers to synthesise the lambda function $\lambda X Y$. $X(X Y)$. Despite its simple nature, no current first-order prover would be able to solve the problem unless provided with the definition of the Church numeral 2.

An option is to convert combinatory terms used in the first-order prover into $\lambda$-terms at the point of unification and then run a HOU algorithm on these terms. However, the usage of $\lambda$-binders adds complications and subtleties to the implementation of higherorder unification. It is precisely to deal with such issues that explicit substitution calculi [12] [10] have been investigated.

First-order provers are generally not able to handle binders, so rather than explicit substitution calculi, we focus on higher-order unification in the setting of combinatory logic. The only existing algorithm in this setting is Dougherty's algorithm. The algorithm is a complete unification procedure for polymorphic higher-order terms. It is unattractive for implementation because it produces many redundant unifiers and does not terminate in many cases.

Contribution Our main contributions in this paper are:

- A modification of Dougherty's algorithm that works on monomorphic higher-order terms (Section 47. Our algorithm is incomplete, but terminating and has shown strong experimental results.

- A method of imperfect filtering that facilitates the implementation of higher-order unification without harming performance on first-order problems (Section 5).

These techniques are implemented in the Vampire theorem prover [17] (along with other extensions reported elsewhere for higher-order reasoning) and experimental results (Section 6) show that combinatory unification can help solve previously unsolved problems.

\section{Preliminaries}

In this paper some knowledge of first-order unification and substitution tree indexing is assumed. The reader is referred to [15] and [14] for further details. We present the log- 
ical terminology used throughout the rest of the paper. We work with the combinatorylogic (CL) first developed by Schönfinkel, but popularised by Curry. As Dougherty's original algorithm works with polymorphic terms and our modification works with monomorphic terms, both are presented here.

Terms are built over a set of types. Let $S$ be a set of sort symbols that act as syntactic identifiers for the base types of the logic and $V_{t y}$ be a set of sort variables. The set of types is defined as:

$$
\begin{array}{lll}
\text { Monomorphic Types } & \tau::=\sigma \mid \tau \rightarrow \tau \quad \text { where } \sigma \in S \\
\text { Polymorphic Types } & \tau::=\sigma|\alpha| \tau \rightarrow \tau \quad \text { where } \sigma \in S, \alpha \in V_{t y}
\end{array}
$$

A polymorphic type declaration is of the form $\Pi \overline{\alpha_{m}} . \tau$ where each $\alpha_{i}$ is a type variable and $\tau$ is a potentially polymorphic type containing type variables from $\overline{\alpha_{m}}$ ( $\overline{\alpha_{m}}$ is a list of type variables). A monomorphic type declaration is simply $\tau$ for some monomorphic type $\tau$.

For each type $\tau$ let $V_{\tau}$ be a set of term variables of type $\tau$ and let $V=\bigcup_{\tau \in T} V_{\tau}$. Further, let $\Sigma$ be a set of typed constant symbols. When working in monomorphic CL, for every type $\tau$, there exists a constant I : $\tau \rightarrow \tau \in \Sigma$. For every pair of types $\tau, \rho$, there exists a constant $\mathbf{K}: \tau \rightarrow \rho \rightarrow \tau \in \Sigma$ and for every triple of types $\tau, \rho, \sigma$, there exists a constant $\mathbf{S}:(\tau \rightarrow \rho \rightarrow \sigma) \rightarrow(\tau \rightarrow \rho) \rightarrow \tau \rightarrow \sigma \in \Sigma$. The constants $\mathbf{I}, \mathbf{K}$ and $\mathbf{S}$ are known as basic combinators. When working in polymorphic CL, the existence of only three polymorphic basic combinators is required. From now on, unless required for clarity, type subscripts are omitted.

We define monomorphic and polymorphic terms together as follows. Let $f: \Pi \overline{\alpha_{m}} . \tau$ be a member of $\Sigma$. In the monomorphic case, $m=0$. Then, $f$ applied to $m$ type arguments: $f\left\langle\overline{\sigma_{m}}\right\rangle$ is a term of type $\tau\left\{\overline{\alpha_{m}} \rightarrow \overline{\sigma_{m}}\right\}$ for some tuple of types $\overline{\sigma_{m}}$. For all $X \in V_{\tau}, X$ is a term of type $\tau$. If $t$ is a term of type $\tau \rightarrow \sigma$ and $t^{\prime}$ is a term of type $\tau$, then $t t^{\prime}$ is a term of type $\sigma$. Where type arguments are irrelevant, they are dropped from the presentation.

Terms of the form $t t^{\prime}$ are called applications. Non-applicative terms $X, \zeta \in V \cup \Sigma$ are called heads. A term can be decomposed uniquely into a head and $n$ arguments, e.g. $\zeta t_{1} \ldots t_{n}$ or in shorter form $\zeta \overline{t_{n}}$. By head $(t)$ the unique head of $t$ is intended, e.g. head $(f a b)=f$. A head is first-order if it is not a variable or combinator. A term is passive if it does not have a combinator head. The positions $\operatorname{pos}(t)$ of term $t$ are defined in the standard fashion; we write $\left.t\right|_{p}$ for the subterm of $t$ at position $p$. Recall the partial ordering $<$ on positions such that $p<p^{\prime}$ if $\left.t\right|_{p^{\prime}}$ is a subterm of $\left.t\right|_{p}$. The set of all positions over $t$ is denoted $\operatorname{pos}(t)$ and the size of $t$, denoted $|t|$, is the cardinality of $\operatorname{pos}(t)$. A higher-order subterm 1 is a subterm with a variable or combinator head. A term that contains no higher-order subterms is called first-order. The set of first-order positions over a term $t$ is defined as all $p \in \operatorname{pos}(t)$ such that, for all $p^{\prime}<p$, head $\left(\left.t\right|_{p^{\prime}}\right)$ is not a variable or combinator. A term is linear if it contains no repeated variables. In a term $\zeta \overline{t_{n}}$, subterms of the form $\zeta \overline{t_{i}}$ for $i<n$ are known as prefix subterms.

In what follows, capital letters such as $X, Y, Z \ldots$ are used to denote variables, $s, t, u$ denote arbitrary terms, $a, b, c \ldots$ denote constants.

\footnotetext{
${ }^{1}$ Note that the definition of higher-order subterm here is different to its usage in [3]
} 
Unification Unification involves substituting terms for (free) variables in order to make two or more terms equal. This equality could be syntactic equality, as is generally the case in first-order theorem proving, or equality modulo a set of axioms. In classic HOU, the goal is to find substitution(s) $\theta_{1} \ldots \theta_{n}$ for terms $t_{1}, t_{2} \ldots t_{n}$ such that $t_{1} \theta_{i}={ }_{\beta \eta}$ $t_{2} \theta_{i}={ }_{\beta \eta} \ldots={ }_{\beta \eta} t_{n} \theta_{i}$ where $=_{\beta \eta}$ is equality modulo the axioms of $\beta$ and $\eta$ reduction. In this paper, we are interested in the relationship $={ }_{c}$ (defined below) on terms of the combinatory logic. A substitution that unifies two or more terms is known as a unifier. When working with polymorphic terms, a substitution $\theta$ is a pair, a term substitution $\theta_{0}$ and a type substitution $\theta_{1}$. By an abuse of notation, the same symbols are used to refer to these dual substitutions and standard monomorphic substitutions.

For two unifiers $\sigma$ and $\theta, \sigma$ is more general than $\theta(\sigma \leq \theta)$ iff there exists a substitution $\gamma$ such that $\sigma \gamma=\theta$. In this case, $\theta$ is redundant. If neither $\sigma \leq \theta$, nor $\theta \leq \sigma$ then $\sigma$ and $\theta$ are independent. In syntactic first-order unification, if two terms have a unifier then they have a unique (up to variable naming) most general unifier (mgu). This is not the case with HOU and the notion of mgu is generalised to that of complete set of unifiers (csu). Let $\Gamma$ be a set of unifiers of terms $t_{1} \ldots t_{n} . \Gamma$ is a csu iff for all $\sigma \in$ unifiers $\left(\overline{t_{n}}\right)$ such that $\sigma \notin \Gamma$, we have $\exists \sigma^{\prime} \in \Gamma$ such that $\sigma^{\prime} \leq \sigma$. $\Gamma$ is a minimal csu iff for all $\sigma_{1}, \sigma_{2} \in \Gamma, \sigma_{1}$ and $\sigma_{2}$ are independent. With respect to HOU, all minimal csus may be infinite.

The HOU problem is undecidable and any complete algorithm must produce redundant unifiers [16]. Let $\stackrel{u p}{=}$ be the least congruence relation on combinatory terms which contains $\left\{\left(t_{1}, t_{2}\right) \mid\right.$ head $\left(t_{1}\right)$, head $\left.\left(t_{2}\right) \in V\right\}$. The pre-unification problem is to find substitutions $\theta_{1} \ldots \theta_{n}$ such that for terms $t_{1} \ldots t_{n}, t_{i} \theta_{k} \stackrel{u p}{=} t_{j} \theta_{k}$ for all $i, j$ and $k$. Huet devised a famous complete algorithm for pre-unification [16] that is irredundant.

Definition 1. For $C L$ terms $t_{1}$ and $t_{2}, t_{1}={ }_{c} t_{2}$ or equivalently $t_{1}$ is $C$-equal to $t_{2}$, iff $\Lambda\left(t_{1}\right)={ }_{\beta \eta} \Lambda\left(t_{2}\right)$ where $\Lambda$ is the following translation between combinatory terms and terms of the $\lambda$-calculus.

$$
\begin{array}{ll}
\Lambda(a)=a \text { for a not a combinator } & \Lambda(\mathbf{S})=\lambda X Y Z . X Z(Y Z) \\
\Lambda(\mathbf{I})=\lambda X . X & \Lambda\left(t_{1} t_{2}\right)=\Lambda\left(t_{1}\right) \Lambda\left(t_{2}\right) \\
\Lambda(\mathbf{K})=\lambda X Y . X &
\end{array}
$$

The translation $\Lambda$ can be used to derive unifiers of $\lambda$-terms from unifiers of CL terms. The details can be found in Dougherty's paper [11]. If $\theta$ is a substitution such that $\theta$ unifies two or more terms with respect to ${ }_{c}, \theta$ is referred to as a C-unifier.

Following Dougherty, a system is defined as a multiset of pairs of CL terms. A pair is trivial if its components are identical and it is valid if its components are $\mathrm{C}$-equal. The definition of flex-flex, flex-rigid and rigid-rigid pairs is as common in HOU literature [27]. The definitions of trivial and valid are extended to systems in the obvious way. The extensional combinatory unification problem is to find, for any given system $\mathcal{S}$, a set of unifiers $U$ such that $\forall\left\langle t_{1}, t_{2}\right\rangle \in \mathcal{S}$ and $\forall \theta \in U t_{1} \theta={ }_{c} t_{2} \theta$. A system $\mathcal{S}$ is simple if for all pairs $\left\langle t, t^{\prime}\right\rangle$ in $\mathcal{S}$, terms $t$ and $t^{\prime}$ do not have identical rigid heads and both are passive.

Definition 2 (Solved System). For some system $\mathcal{S}$, a pair $\left\langle X, t_{2}\right\rangle \in \mathcal{S}$ is solved if $X$ doesn't occur in $t_{2}$ or in any other pair in $\mathcal{S}$. A system $\mathcal{S}$ is solved if $\forall p \in \mathcal{S}$, either $p$ is trivial or solved. 
The importance of the concept of solved systems can be seen from the fact that the solved pairs of a solved system $\mathcal{S}$ form a most general unifier of $\mathcal{S}$ (see [11]).

\section{Dougherty's Combinatory Unification Algorithm}

Dougherty's algorithm is a complete, finitely-branching, polymorphic HOU algorithm. It is presented here as a set of non-determinsitic transformation rules that act on a system of unification pairs. Let $\Longrightarrow$ represent the application of a transformation rule to a system, $\Longrightarrow+$ the transitive closure of $\Longrightarrow$ and $\Longrightarrow$ its reflexive transitive closure. We use $\uplus$ for the multiset sum of two multisets. It assumed that the order of the terms in the pairs is immaterial.

Polymorphism is an essential feature in Dougherty's algorithm even if the initial terms are monomorphic. In the SXX'-narrow transform (given below) an applied variable head is replaced with the term $\mathbf{S} X^{\prime} X^{\prime \prime}$ and then reduced. Applying this transform to the system $\left\{\left\langle X_{\iota \rightarrow \iota} a_{\iota}, b_{\iota}\right\rangle\right\}$ results in $\left\{\left\langle X^{\prime} a\left(X^{\prime \prime} a\right), b\right\rangle\right\}$. Because the type of $a$ is $\iota$ the type of the fresh variable $X^{\prime \prime}$ must be $\iota \rightarrow$ ?, but there is no way to determine what type ? should be. Similarly the type of $X^{\prime}$ must be $\iota \rightarrow ? \rightarrow \iota$, but again ? cannot be determined. Thus, in both cases ? is set to a type variable which may be instantiated during subsequent unification. In only this transformation are the types of the introduced variables not deducible.

Our presentation differs from that of Dougherty's by having WEAKREDUCE as a separate transformation, rather than a special case of HEADNARROW.

1. ADDARG

$$
\left\{\left\langle t_{1}, t_{2}\right\rangle\right\} \uplus \mathcal{S} \Longrightarrow\left\{\left\langle t_{1} \theta d, t_{2} \theta d\right\rangle\right\} \uplus \mathcal{S} \theta
$$

Where either $t_{1}$ or $t_{2}$ has an under applied combinator as its head, $d$ is a fresh constant and $\theta$ is the type-unifier of the types of $t_{1}, t_{2}$ and $\alpha \rightarrow \tau$ for fresh type variables $\alpha$ and $\tau$ (in case $t_{1}$ and $t_{2}$ are both of atomic type).

2. SPLiT:

$$
\left\{\left\langle X \overline{t_{n}}, h \overline{s_{m}^{\prime}} \overline{s_{n}^{\prime \prime}}\right\rangle\right\} \uplus \mathcal{S} \Longrightarrow \quad\left\{\left\langle h \overline{X_{m}^{\prime}} \overline{t_{n}}, h \overline{s_{m}^{\prime}} \overline{s_{n}^{\prime \prime}}\right\rangle\right\} \theta \uplus \mathcal{S} \theta
$$

Where $\theta=\left(\theta_{0}, \theta_{1}\right), \theta_{0}=\left\{X \rightarrow h \overline{X_{m}^{\prime}}\right\}$ and $\theta_{1}$ is the mgu of type $(X)$ and $\operatorname{type}\left(h \overline{s_{m}^{\prime}}\right)$. Each $X_{i}^{\prime}$ is a fresh variable.

3. WEAKREDUCE:

$$
\begin{array}{lll}
\left\{\left\langle\mathbf{I} t \overline{t_{n}}, s^{\prime}\right\rangle\right\} \uplus \mathcal{S} & \Longrightarrow\left\{\left\langle t \overline{t_{n}}, s^{\prime}\right\rangle\right\} \uplus \mathcal{S} & \text { (I-reduce) } \\
\left\{\left\langle\mathbf{K} t t^{\prime} \overline{t_{n}}, s^{\prime}\right\rangle\right\} \uplus \mathcal{S} & \Longrightarrow\left\{\left\langle t \overline{t_{n}}, s^{\prime}\right\rangle\right\} \uplus \mathcal{S} & \text { (K-reduce) } \\
\left\{\left\langle\mathbf{S} t t^{\prime} t^{\prime \prime} \overline{t_{n}}, s^{\prime}\right\rangle\right\} \uplus \mathcal{S} & \Longrightarrow\left\{\left\langle t t^{\prime \prime}\left(t^{\prime} t^{\prime \prime}\right) \overline{t_{n}}, s^{\prime}\right\rangle\right\} \uplus \mathcal{S} & \text { (S-reduce) }
\end{array}
$$

4. HeadNarRow: The variables introduced by the rules are assumed to be fresh for the system in all cases. In all rules, $\theta=\left(\theta_{0}, \theta_{1}\right)$ where $\theta_{0}$ is the syntactic unifier of a non-variable prefix subterm and the left-hand side of a suitably renamed 
combinator axiom and $\theta_{1}$ is the relevant type unifier. For example, in the first rule $\theta_{0}=\{X \rightarrow \mathbf{I}\}$ and $\theta_{1}=\operatorname{mgu}(\operatorname{type}(X), \operatorname{type}(\mathbf{I}))$.

$$
\begin{array}{lll}
\left\{\left\langle X t \overline{t_{n}}, s^{\prime}\right\rangle\right\} \uplus \mathcal{S} & \Longrightarrow\left\{\left\langle t \overline{t_{n}}, s^{\prime}\right\rangle\right\} \theta \uplus \mathcal{S} \theta \\
\left\{\left\langle X t \overline{t_{n}}, s^{\prime}\right\rangle\right\} \uplus \mathcal{S} & \Longrightarrow\left\{\left\langle X^{\prime} \overline{t_{n}}, s^{\prime}\right\rangle\right\} \theta \uplus \mathcal{S} \theta \quad \text { (I-narrow) } \\
\left\{\left\langle X t t^{\prime} \overline{t_{n}}, s^{\prime}\right\rangle\right\} \uplus \mathcal{S} & \Longrightarrow\left\{\left\langle t \overline{t_{n}}, s^{\prime}\right\rangle\right\} \theta \uplus \mathcal{S} \theta & \text { (KX-narrow) } \\
\left\{\left\langle X t \overline{t_{n}}, s^{\prime}\right\rangle\right\} \uplus \mathcal{S} & \Longrightarrow\left\{\left\langle X^{\prime} t\left(X^{\prime \prime} t\right) \overline{t_{n}}, s^{\prime}\right\rangle\right\} \theta \uplus \mathcal{S} \theta & \text { (K-narrow) } \\
\left\{\left\langle X t t^{\prime} \overline{t_{n}}, s^{\prime}\right\rangle\right\} \uplus \mathcal{S} & \Longrightarrow\left\{\left\langle X^{\prime} t^{\prime}\left(t t^{\prime}\right) \overline{t_{n}}, s^{\prime}\right\rangle\right\} \theta \uplus \mathcal{S} \theta \quad \text { (SX-narrow) } \\
\left\{\left\langle X t t^{\prime} t^{\prime \prime} \overline{t_{n}}, s^{\prime}\right\rangle\right\} \uplus \mathcal{S} & \Longrightarrow\left\{\left\langle t t^{\prime \prime}\left(t^{\prime} t^{\prime \prime}\right) \overline{t_{n}}, s^{\prime}\right\rangle\right\} \theta \uplus \mathcal{S} \theta \quad \text { (S-narrow) }
\end{array}
$$

These four transformation rules are collectively known as the HUT-transformations. They are used alongside syntactic transformations DECOMP, ELIMINATE and TYPEUNIFY. For a system $\mathcal{S}$, its derived system is the system of type pairs formed by replacing each term in $\mathcal{S}$ with its type.

\section{DECOMP:}

$$
\left\{\left\langle f \overline{t_{n}}, f \overline{s_{n}}\right\rangle\right\} \uplus \mathcal{S} \Longrightarrow\left\{\left\langle t_{1}, s_{1}\right\rangle \ldots\left\langle t_{n}, s_{n}\right\rangle\right\} \uplus \mathcal{S}
$$

6. ELIminate:

$$
\{\langle X, t\rangle\} \uplus \mathcal{S} \Longrightarrow\{\langle X, t\rangle\} \uplus \mathcal{S} \theta
$$

where $\theta=\{X \rightarrow t\}$ and $X$ does not occur in $t$.

7. TYPEUNIFY

$$
\mathcal{S} \Longrightarrow \mathcal{S} \theta
$$

Where $\theta$ is the most general type unifier of the derived system of $\mathcal{S}$.

For any system $\mathcal{S}$, an exhaustive application of WEAKREDUCE, DECOMP and ADDARG results in a simple system $\mathcal{S}^{\prime}$ with the same unifiers as $\mathcal{S}$ as proved by Dougherty. Dougherty [11] proves the following non-deterministic algorithm, called $U$, for enumerating $C$-unifiers to be sound and complete.

1. Reduce the system to a simple system then apply some HUT-transformation out of an unsolved pair.

2. If at any point the system is syntactically unifiable by a pure substitution then optionally return a most general unifier of the system.

A substitution $\sigma$ is pure if $\forall x \in \operatorname{dom}(\sigma), \sigma(x)$ does not contain any constants introduced by ADDARG. Unfortunately $U$ contains infinite computation paths in many cases where Huet's classical algorithm does terminate. Worse, even when restricted to pre-unification, the algorithm produces redundant unifiers. 
Lemma 1. If, for a system of unification pairs $\Sigma$, there exists a computation path of $U$ that includes a HEADNARROW step, then there exists an infinite computation path of $U$ on $\mathcal{S}$.

Proof. Let $t y_{1}$ and $t y_{2}$ be meta-type variables, standing for arbitrary types. We show that if a pair of the form $\left.\left\langle X_{t y_{1} \rightarrow t y_{2}} t_{t y_{1}} \overline{t_{n}}, t^{\prime}\right\rangle \quad{ }^{*}\right)$ is part of a simple system, then an SXX'-narrow step can be applied and the resulting system transformed to a simple system containing a pair of the same form.

For the application of a HEADNARROw step, there must exist a simple system $\mathcal{S}_{1}$ such that $\mathcal{S} \Longrightarrow{ }^{*} \mathcal{S}_{1}$ by a series of $U$-steps and $\mathcal{S}_{1}$ includes a pair $p=\left\langle X_{\alpha \rightarrow \tau} t_{\alpha} \overline{t_{n}}, t^{\prime}\right\rangle$. Assume that $t^{\prime}$ has a rigid head or a flexible head different to $X$ (if the head of $t^{\prime}$ is $X$, the proof still holds, but is slightly more complex). The pair $p$ is of the form $(*)$. The following HEADNARROW step can then be applied:

$$
\mathcal{S}_{1}=p \uplus \mathcal{S}_{2} \Longrightarrow_{S X X^{\prime}-\text { narrow }}\left\{\left\langle X_{\alpha \rightarrow \gamma \rightarrow \tau}^{\prime} t_{\alpha}\left(X_{\alpha \rightarrow \gamma}^{\prime \prime} t_{\alpha}\right) \overline{t_{n}}, t^{\prime}\right\rangle\right\} \theta \uplus \mathcal{S}_{2} \theta=\mathcal{S}_{3}
$$

The algorithm proceeds by reducing $\mathcal{S}_{3}$ to a simple system $\mathcal{S}_{4}$. As head $\left(t^{\prime}\right) \neq X$, we have that head $\left(t^{\prime}\right)=$ head $\left(t^{\prime} \theta\right)$. Since neither $X^{\prime}$ nor head $\left(t^{\prime}\right)$ is a combinator, no WEAKREDUCE or ADDARG rules can be applied to the pair during the reduction phase. As $X^{\prime}$ is not rigid, DECOMP is not applicable either. Therefore, the pair $\left\langle\left(X_{\alpha \rightarrow \gamma \rightarrow \tau}^{\prime} t_{\alpha}\left(X^{\prime \prime} t\right) \overline{t_{n}}\right) \theta \sigma, t \theta \sigma\right\rangle$ is a part of $\mathcal{S}_{4}$ where $\sigma$ is a possibly empty type substitution introduced by ADDARG steps. By taking $t y_{1}=\alpha \theta \sigma$ and $t y_{2}=(\gamma \rightarrow \tau) \theta \sigma$, we have that $\mathcal{S}_{4}$ is a simple system that contains a pair of form (*).

Lemma 2. Even if $U$ is restricted, such that no transformation steps are carried out on flex-flex pairs, $U$ can still produce redundant unifiers.

Proof. Consider the simple system $\{\langle X a, a\rangle\}$. Then by a single application of I-narrow the unifier $\{X \rightarrow \mathbf{I}\}$ can be produced. Alternatively the derivation path $\{\langle X a, a\rangle\}$ $\Longrightarrow_{S X X^{\prime}-\text { narrow }}\left\{\left\langle X^{\prime} a\left(X^{\prime \prime} a\right), a\right\rangle\right\} \Longrightarrow_{K \text {-narrow }} \Longrightarrow\{\langle a, a\rangle\}$ can be followed leading to the unifier $\left\{X \rightarrow \boldsymbol{S} \boldsymbol{K} X^{\prime \prime}\right\} . \boldsymbol{S} \boldsymbol{K} X^{\prime \prime}{ }_{c_{c}} \mathbf{I}$ and is thus a redundant unifier.

Lemmas 1 and 2 show that Dougherty's algorithm, whilst interesting from a theoretical aspect, is not suitable for a practical implementation. In as yet unpublished work [4], Bentkamp et al. present a modification of the given-clause algorithm that deals with possibly infinite streams of unifiers. But even such a method would be unable to handle Dougherty's algorithm as almost all unification problems are likely to be non-terminating quickly leading to memory issues on difficult problems. Instead, we propose a modification to the algorithm that eliminates these unpleasant properties at the cost of completeness.

\section{Restricted Combinatory Unification}

The pair of problems with Dougherty's algorithm identified in the previous section are both linked to the SXX'-narrow transform. As this step introduces type variables, typing cannot be used to restrict its application. In our modification of Dougherty's algorithm, we remove this head-narrow step. As this step was the only one to introduce type variables, polymorphism can now be eliminated. The three polymorphic combinator axioms 
used in the HEADNARROW step now become an infinite set of monomorphic axioms. To this set the $\mathbf{C}$ and $\mathbf{B}$ combinator axioms schemas are added. These schemas are $\mathbf{B} X Y Z=X(Y Z)$ and $\mathbf{C} X Y Z=X Z Y$. The $\mathbf{C}$ and $\mathbf{B}$ combinators are redundant, in the sense that they can be defined in terms of $\mathbf{S}, \mathbf{K}$ and $\mathbf{I}$, yet their usage often makes combinatory terms smaller.

Below, the modifications to Dougherty's algorithm are presented. The resulting algorithm is referred to as Restricted Combinatory Unification or RCU. In the calculation of the unifier $\theta$ in the steps below, no type unification is required. Further Dougherty's syntactic transformation step TYPEUNIFY is no longer required resulting in DECOMP and ELIMINATE being the only syntactic transforms needed.

Definition 3. The set of all variables contained in a system $\mathcal{S}$, denoted vars $(\mathcal{S})$, is divided into two disjoint subsets $R$ and $B$. Members of $R$ are referred to as red variables and members of $B$ as blue variables. We define $R=\{X \in \operatorname{vars}(\mathcal{S}) \mid X$ introduced by $C X$-narrow transform $\}$ and $B=\operatorname{vars}(\mathcal{S})-R$.

1. The following WeakReducE rules are added in addition to the three in Dougherty's algorithm.

$$
\begin{array}{lll}
\left\{\left\langle\mathbf{B} t t^{\prime} t^{\prime \prime} \overline{t_{n}}, s^{\prime}\right\rangle\right\} \uplus \mathcal{S} & \Longrightarrow\left\{\left\langle t\left(t^{\prime} t^{\prime \prime}\right) \overline{t_{n}}, s^{\prime}\right\rangle\right\} \uplus \mathcal{S} & \text { (B-reduce) } \\
\left\{\left\langle\mathbf{C} t t^{\prime} t^{\prime \prime} \overline{t_{n}}, s^{\prime}\right\rangle\right\} \uplus \mathcal{S} & \Longrightarrow\left\{\left\langle t t^{\prime \prime} t^{\prime} \overline{t_{n}}, s^{\prime}\right\rangle\right\} \uplus \mathcal{S} \quad \text { (C-reduce) }
\end{array}
$$

2. The SXX'-narrow step is removed from Dougherty's rules and the following HEADNARROW steps are added. In all cases, $\theta$ is the syntactic (first-order) unifier of a non-variable prefix subterm and the left-hand side of a suitably renamed combinator axiom.

$$
\begin{array}{lll}
\left\{\left\langle X t t^{\prime} \overline{t_{n}}, s^{\prime}\right\rangle\right\} \uplus \mathcal{S} & \Longrightarrow\left\{\left\langle X^{\prime}\left(t t^{\prime}\right) \overline{t_{n}}, s^{\prime}\right\rangle\right\} \theta \uplus \mathcal{S} \theta \quad \text { (BX-narrow) } \\
\left\{\left\langle X t t^{\prime} t^{\prime \prime} \overline{t_{n}}, s^{\prime}\right\rangle\right\} \uplus \mathcal{S} & \Longrightarrow\left\{\left\langle t\left(t^{\prime} t^{\prime \prime}\right) \overline{t_{n}}, s^{\prime}\right\rangle\right\} \theta \uplus \mathcal{S} \theta \quad \text { (B-narrow) } \\
\left\{\left\langle X t t^{\prime} \overline{t_{n}}, s^{\prime}\right\rangle\right\} \uplus \mathcal{S} & \Longrightarrow\left\{\left\langle X^{\prime} t^{\prime} t \overline{t_{n}}, s^{\prime}\right\rangle\right\} \theta \uplus \mathcal{S} \theta \quad \text { (CX-narrow) }
\end{array}
$$

Where $X$ is not a red variable

$$
\left\{\left\langle X t t^{\prime} t^{\prime \prime} \overline{t_{n}}, s^{\prime}\right\rangle\right\} \uplus \mathcal{S} \quad \Longrightarrow\left\{\left\langle t t^{\prime \prime} t^{\prime} \overline{t_{n}}, s^{\prime}\right\rangle\right\} \theta \uplus \mathcal{S} \theta \quad \text { (C-narrow) }
$$

The reason for the restriction on the CX-narrow step is to prevent infinite computation paths such as $\langle X a b, s\rangle \Longrightarrow\left\langle X^{\prime} b a, s\right\rangle \Longrightarrow\left\langle X^{\prime \prime} a b, s\right\rangle \Longrightarrow \ldots$. The convention that no transformations are carried out on solved or trivial pairs is adopted.

Restricting Dougherty's algorithm would be of little interest if the restricted version suffered the same problems as the original. This is not the case with RCU.

Theorem 1. Every sequence of RCU transformations terminates 
Proof. A proof sketch is provided here (see Appendix A for full proof). Dougherty proves that the set of transforms WEAKREDUCE, DECOMP and ADDARG are terminating. We reduce the proof of termination of RCU to this proof by showing that the remaining transformation can only appear finitely many times on a computation path. Intuitively, for any type $\sigma$, size $(\sigma)$ is the number of ' $\rightarrow$ 's in $\sigma$.

- Each of transforms ELIMINATE and SPLIT, reduce the number of unsolved variables in a system by 1 , whilst all other transforms maintain or reduce this measure.

- For HeAdNARRow, consider the measure $\left(\sum_{v \in \text { vars }(\mathcal{S})}\right.$ size $($ type $\left.(v)), \# \mathcal{S}\right)$ where $\# \mathcal{S}$ is the number of blue variables in a system $\mathcal{S}$. HEADNARROW reduces this measure whilst WEAKREDUCE, DECOMP and ADDARG maintain or reduce it.

An obvious corollary of Theorem 1 is that RCU is not a complete HOU algorithm. It does not, in general, find a csu. It would be of interest to compare RCU with other restricted forms of higher-order unification such as pattern unification [22]. However, it is not readily comparable with pattern unification and similar restrictions, as these tend to be restrictions on the input terms whilst RCU is a restriction on the transformations. As such, it most closely resembles depth-bound version of Huet's algorithm. We have not studied the complexity of RCU, but in Section 6, empirical evidence is presented that suggests its performance is reasonable.

\section{$5 \quad$ Imperfect Filtering}

In first-order provers, unification is generally carried out via term-indexes. Let $R$ be a relationship on terms. A term-indexing data-structure stores terms in a manner that facilitates the rapid retrieval of all terms $l$ such that $R(l, t)$ for some query term $t$. In the context of theorem-proving, the relationship $R$ could be "is unifiable with", "is a generalisation of" etc. Indexing structures for first-order theorem proving have been intensively studied and efficient indexing structures such as substitution trees, fingerprint indexes and perfect discrimination trees have been developed.

These structures are either perfect or imperfect depending on whether they return all and only those terms that match the query, or they return some sub/superset of the same. Substitution trees store substitutions in their nodes. Variables of the form $*_{i}$ are used to denote substitutions in the tree. The equation $*_{i}=t$ represents the substitution of the variable $*_{i}$ by the term $t$. A path from the root to a leaf represents a term formed by the composition of substitutions on the path (view Figure 1). Substitution trees act as perfect filters for first-order unification. This is achieved by traversing the tree left-right depth-first. In the root, the query term is unified with $*_{0}$. Each time we move down to a node $*_{i}=t$ the current unifier is extended with the unifier of $\left(*_{i}, t\right)$ in what is known as incremental unification. On backtracking, the unifier is reset to its previous value.

With respect to higher-order unification, some work has been carried out on developing indexing data structures [20] [24], but has gained little acceptance. Steen [28] suggests that the reason for this is the complexity or undecidability of many of the operations required to build and maintain higher-order indexing structures. For example, higher-order unification, anti-unification and matching are all either undecidable or have large complexities. This is a daunting obstacle to developing perfect higher-order indexing structures. However, it does not preclude the development of imperfect filters. 
We have modified substitution trees to act as imperfect filters for higher-order unification. The insights behind this modification are two-fold:

1. If two terms disagree on a function symbol that is not below an applied variable or combinator, then the terms have no higher-order unifier

2. Two terms that are first-order can only have a first-order unifier

Description of Filtering Algorithm Prior to any term being inserted into the tree all higher-order subterms are replaced with special sort-correct constants $\#_{\tau}$ not appearing in the input. We call this process hashing and its inverse de-hashing. When performing incremental unification, '\#'s unify with all terms. Let $t$ be a query term and $T$ a hashed substitution tree. The filtering algorithm works as follows:

1. Calculate $t^{\prime}=\operatorname{hash}(t)$.

2. Run the standard first-order algorithm to find all unification partners of $t^{\prime}$ in $T$. Let $U=\left\{\left\langle\sigma_{i}, t_{i}\right\rangle \mid t_{i}\right.$ unifies with $t^{\prime}$ with unifier $\left.\sigma_{i}\right\}$ be the output of this algorithm.

3. For all $\left\langle\sigma_{i}, t_{i}\right\rangle$ in $U$, if both $t$ and dehash $\left(t_{i}\right)$ are first-order then return $\sigma_{i}$ as the only unifier of $t$ and $\operatorname{deh} a s h\left(t_{i}\right)$.

4. Otherwise the pair $\left\langle t, t_{i}\right\rangle$ has passed filtering and is handed over to RCU.

Correctness of Algorithm To show that the algorithm is correct, we need to show that (1) if incremental unification at a node $*_{i}=t$ fails due to CLASH or OCCURSCHECK (terminology from [1]), then for all terms sharing this substitution, none of them can have a higher-order unifier with the query term. We also need to show that (2) if two terms are first-order then higher-order unification can only produce the first-order unifier. The following two lemmas are used to show (1).

Lemma 3. If $\left.t\right|_{p}=s$ for first-order position $p$, then $\left(\left.t \theta\right|_{p}\right)=s \theta$ for all substitutions $\theta$.

Proof. Proof is by induction on the length of $p$. If $p=\epsilon$ then $\left.t \theta\right|_{\epsilon}=t \theta=s \theta$. In the inductive case, $\left.t \theta\right|_{p}=\left.\left.t \theta\right|_{p^{\prime} . i} \stackrel{I H}{=}\left(\zeta s_{1}^{\prime} \ldots s_{i-1}^{\prime}, s, s_{i+1}^{\prime} \ldots s_{n}^{\prime}\right) \theta\right|_{i}=\left(\zeta s_{1}^{\prime} \theta \ldots\right.$ $\left.s_{i-1}^{\prime} \theta, s \theta, s_{i+1}^{\prime} \theta \ldots s_{n}^{\prime} \theta\right)\left.\right|_{i}=s \theta$.

Lemma 4. Let $p$ be a first-order position in terms $t_{1}$ and $t_{2}$. Then $t_{1}$ and $t_{2}$ have no higher-order unifiers if:

1. Both head $\left(\left.t_{1}\right|_{p}\right)$ and head $\left(\left.t_{2}\right|_{p}\right)$ are first order and head $\left(\left.t_{1}\right|_{p}\right) \neq$ head $\left(\left.t_{2}\right|_{p}\right)$, or

2. $\left.t_{1}\right|_{p}=X, X$ occurs in $\left.t_{2}\right|_{p}$ at a first-order position and $X \neq\left. t_{2}\right|_{p}$ or vice versa.

Proof. Assume that $\theta$ is a unifier of $t_{1}$ and $t_{2}$. Let $\left.t_{1}\right|_{p}$ be $s_{1}$ and $\left.t_{2}\right|_{p}$ be $s_{2}$. By Lemma 3. $\left(\left.t_{1}\right|_{p}\right) \theta=s_{1} \theta$ and $\left(\left.t_{2}\right|_{p}\right) \theta=s_{2} \theta$. Therefore, we must have that $s_{1} \theta=s_{2} \theta$. In case (1), $s_{1}=f \overline{s_{n}^{\prime}}, s_{2}=g \overline{t_{m}^{\prime}}$ and $f \neq g$. However, $s_{1} \theta=\left(f \overline{s_{n}^{\prime}}\right) \theta=f\left(\overline{s_{n}^{\prime}} \theta\right) \neq$ $g\left(\overline{t_{m}^{\prime}} \theta\right)=\left(g \overline{t_{m}^{\prime}}\right) \theta$ and thus $\theta$ cannot be a unifier. In case (2), $s_{1}=X$ and $s_{2}=\zeta \overline{t_{n}}$ such that for some $t_{i}$ and position $p^{\prime},\left.t_{i}\right|_{p^{\prime}}=X$. By Lemma 3 and the fact $i . p^{\prime}$ is a first-order position in $s_{2}$, we have that $\left.s_{2} \theta\right|_{i . p^{\prime}}=X \theta$. But then we cannot have that $s_{1} \theta=s_{2} \theta$ since $\left|s_{1} \theta\right|=|X \theta| \leq\left|t_{i} \theta\right|<\left|\zeta\left(\overline{t_{n}}\right) \theta\right|=\left|s_{2} \theta\right|$. 
Now consider incremental unification at a node $*_{i}=t$. Assume that $*_{i}$ is bound to a subterm $t^{\prime}$ of the hashed query term. If a CLASH occurs in unifying $t$ with $t^{\prime}$, then the query term and all terms sharing the substitution $*_{i}=t$ must disagree on a head symbol occurring at a first-order position. By Lemma 4, the query term and all terms sharing the substitution $*_{i}=t$ can have no higher-order unifiers. The case where unification of $t$ and $t^{\prime}$ fails due to OCCURSCHECK is similar.

To show (2) we prove the following lemma.

Lemma 5. Let $t_{1}$ and $t_{2}$ be first-order terms. Then, for any computation path $\left\{\left\langle t_{1}, t_{2}\right\rangle\right\}$ ${ }^{*} \mathcal{S}^{\prime}$, we have:

1. All terms in $\mathcal{S}^{\prime}$ are first-order.

2. In the computation path, only DECOMP and ELIMINATE are used.

Proof. Proof by induction on the length of the $\Longrightarrow$ * path. The base case is trivial. In the inductive case, after $p$ steps the original system is transformed into $\left\{\left\langle s_{1}, s_{2}\right\rangle \ldots\left\langle s_{n-1}, s_{n}\right\rangle\right\}$ where each $s_{i}$ is first-order by the induction hypothesis. In the $p+1$ step, an arbitrary pair $\left\langle s_{i}, s_{i+1}\right\rangle$ is transformed. If either $s_{i}$ or $s_{i+1}$ is a variable than the transformation is ELIMINATE and the resulting system again contains only first-order terms. Otherwise $s_{i}$ and $s_{i+1}$ are of the form $\zeta \overline{t_{m}}$ and $\zeta \overline{r_{m}}$ and the only applicable step is DECOMP. After performing DECOMP, the resulting system is $\left\{\left\langle s_{1}, s_{2}\right\rangle \ldots \overline{\left\langle t_{m}, r_{m}\right\rangle} \ldots\left\langle s_{n-1}, s_{n}\right\rangle\right\}$ and again all terms are first-order.

Since the usage of DECOMP and EliminATE is deterministic, and these two transforms form a sound and complete unification algorithm for syntactic first-order unification [1], we have that if two terms are first-order and unifiable then running RCU on the terms will result in the single mgu produced by first-order unification. The result is presented in terms of RCU, but is in reality general.

Example Consider searching the tree in Figure 1 for unifiers of $g(f X) b$. The original substitution is $\sigma_{0}=\left\{*_{0} \rightarrow g(f X) b\right\}$. In the root, the substitution is extended to unify $*_{0}$ with $g *_{1} *_{2}$ resulting in $\sigma_{1}=\sigma_{0} \cup\left\{*_{1} \rightarrow f X, *_{2} \rightarrow b\right\}$. In the left child, $*_{1}$ is unified with \#. This succeeds without adding anything to the unifier, so $\sigma_{3}=\sigma_{2}$. Finally, in the left-most leaf, $*_{2}$ is unified with $b$, again succeeding with the empty substitution. The search then backtracks and attempts to enter the second-to-left leaf. This requires unifying $*_{2}$ which is bound to $b$, with $c$ and fails due to CLASH. The terms $g(f X) b$ and $g(\mathbf{I} a) c$ can have no unifiers as symbols $b$ and $c$ which are not below a variable or combinator disagree. The set of terms eventually returned by the query is $\{g(X a) b, g(f a) b, g(f(Z d)) b\}$. As both $g(f X) b$ and $g(f a) b$ are first-order, RCU does not need to run on this pair. RCU is run on the pairs $\langle g(f X) b, g(X a) b\rangle$ and $\langle g(f X) b, g(f(Z d)) b\rangle$

Note that if the input problem is first-order, then all terms in the index and all query terms will be first-order. Thus, in this case, unification will always be first-order unification, and the addition of RCU to Vampire is graceful in the sense of [3].

\section{Experimental Results}

As with most successful first-order provers, Vampire is a portfolio prover. In finding a proof, it runs a set of strategies known as a schedule. Each strategy is a predefined set of 


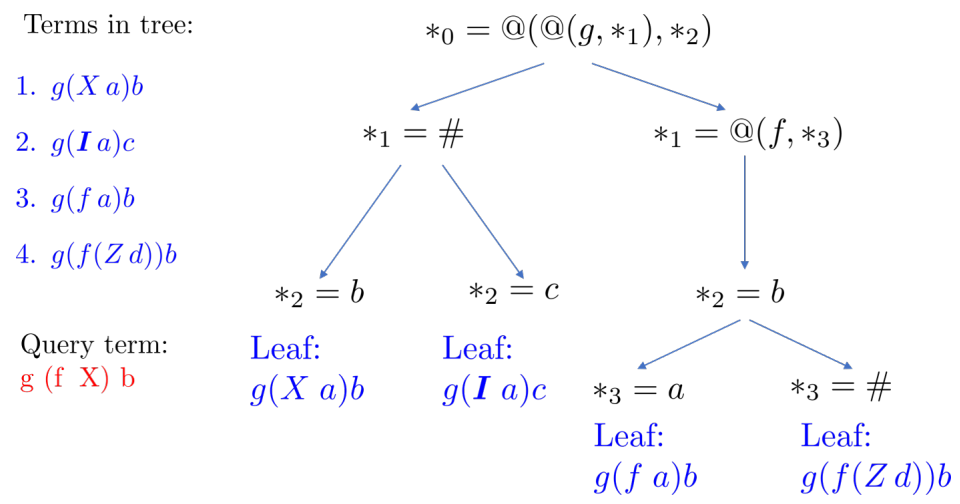

Fig. 1: Example of substitution tree being used as a filter for higher-order unification. The query will return terms (1),(3) and (4). Higher-order unification only needs to be run on the pairs $\langle g(f X) b, g(X a) b\rangle$ and $\langle g(f X) b, g(f(Z d)) b\rangle$

proof search parameters. Normally, if a problem is solvable, it is solvable within a short space of time using a particular strategy. In this section we examine whether the new RCU option can be used to complement the existing set of options available for higherorder reasoning in Vampire. To test this we first heuristically created a custom higherorder schedule that includes various options (including RCU) linked to higher-order proof search. Some details regarding these options can be found in [5]. Two options in particular are relevant to the experiments below:

comb_unif: this option can be set to on or off toggling combinatory unification as described in this paper

combinatorelimination: this option can be set to axioms in which case combinators are axiomatised. It can be set to inference_rules to enable a set of inferences that rewrite fully applied combinators. Axioms and rewriting can be used together by setting the option to both or the option can be set to of $f$

The higher-order schedule represents our current best-effort prior to these experiments e.g. it is Vampire trying its hardest rather than a default baseline.

Experiments were run across two benchmark sets. The first set consists of TPTP library benchmarks [31]. From the TPTP library we selected higher-order monomorphic problems that are designated as theorems, unsatisfiable or unknown giving a total of 2727 problems. Satisfiable problems were excluded as both Vampire and Leo-III are incomplete. The second benchmark set was produced by the Isabelle theorem prover's Sledgehammer system. It contains 1253 benchmarks kindly made available to us by the Matryoshka team and is called $\mathrm{SH}-\lambda$ following their naming convention.

We ran Vampire's higher-order schedule across both benchmark sets multiple times with comb_unif and comb-inator_elimination forced to various values across the whole schedule. A (wall-clock) time limit of 520s was used for experiments over the TPTP problem set and 300s for experiments over the SH- $\lambda$ problem set. Experiments were performed on StarExec [30] nodes equipped with four $2.40 \mathrm{GHz}$ Intel Xeon CPUs. 
Table 1: Number of problems proved theorem or unsat

\begin{tabular}{|c|c|c|c|c|c|c|c|}
\hline & \multicolumn{3}{|c|}{ TPTP problems } & \multicolumn{3}{|c|}{ SH- $\lambda$} \\
\hline & & \multirow{2}{*}{$\begin{array}{l}\text { Number } \\
\text { Solved }\end{array}$} & \multicolumn{2}{|c|}{ Uniques } & \multicolumn{3}{|c|}{ Number Uniques } \\
\hline & & & & A vs B & Solved & All & vs B \\
\hline & Vampire-HOL-RCUon & 1907 & 7 & & 713 & 0 & \\
\hline & Vampire-HOL-RCUon-CEaxs & 1538 & 2 & & 708 & 0 & \\
\hline (A) & Vampire-HOL-RCUon-CEinf & 1728 & 0 & 276 & 714 & 0 & 16 \\
\hline & Vampire-HOL-RCUon-CEoff & 1500 & 0 & & 700 & 0 & \\
\hline & Vampire-HOL-RCUoff & 1920 & 30 & & 725 & 1 & \\
\hline (B) & Vampire-HOL-RCUoff-CEaxs & 1637 & 15 & 175 & 715 & 0 & 17 \\
\hline & Vampire-HOL-RCUoff-CEinf & 1672 & 0 & & 720 & 0 & \\
\hline & Vampire-HOL-RCUoff-CEoff & 1186 & 0 & & 692 & 0 & \\
\hline & Union & 2018 & & & 734 & & \\
\hline & Vampire-HOL & 1958 & 31 & & 719 & 55 & \\
\hline & Leo-III & 2097 & 58 & & 668 & 31 & \\
\hline & Satallax & 2095 & 92 & & 513 & 8 & \\
\hline
\end{tabular}

The results of two sets of experiments can be found in Table 11. In the first set, Vampire-HOL-RCUxx-CEyyy refers to Vampire running its higher-order schedule, with comb_unif forced to value $\mathbf{x x}$ and combinator_elimination forced to value yyy. The second set compares Vampire-HOL (higher-order schedule with nothing forced) to the CASC-2018 versions of leading higher-order theorem provers, LeoIII version 1.3 and Satallax version 3.3 [6]. The number of problems solved uniquely are given separately for the two sets of experiments. As explained below, we make an additional comparison in the first set. Our experimental data is publicly available ${ }^{2}$

Firstly, we consider the choice between reasoning solely with axioms or solely with RCU. To do this we compare Vampire-HOL-RCUon-CEinf (A) and Vampire-HOLRCUoff-CEaxs (B). In (B) the only method of synthesising higher-order functions is via combinators and in (A) it is via RCU, making these suitable versions for comparing the two methods. RCU is run in conjunction with inference rules in our comparison due to the implementation of RCU which does not weak reduce terms on the application of a combinatory unifier. Across the TPTP benchmark set, RCU significantly outperforms axioms. Across the $\mathrm{SH}-\lambda$ problem set, the performance of the two is similar.

Next we consider the other combinations of options more broadly. The strategies that solve the most problems do not force RCU on. Indeed, between them, strategies forcing RCU off solve 93 problems unsolved by forcing RCU on, conversely 68 are solved by forcing it on as opposed to off. This is expected as RCU is expensive and many problems do not require it. It is the 68 problems gained with the new option that are of interest. We also draw attention to the fact that for both problem sets, the union of all problems solved by the various Vampire versions exceeds the number of problems solved by our current schedule. This suggests that the schedule could easily be improved by incorporating some of these strategies.

2 https://github.com/vprover/vampire_publications/tree/master/ experimental_data/CADE-2019-RCU 
Table 2: Efficiency of RCU

\begin{tabular}{lll}
\hline $\begin{array}{l}\text { Problem } \\
\text { Category }\end{array}$ & $\begin{array}{l}\text { Average time spent on } \\
\text { combinatory unification (as \% } \\
\text { of total time) }\end{array}$ & $\begin{array}{l}\text { Average number of unifiers } \\
\text { produced per problem }\end{array}$ \\
\hline SYO & $11.71 \%$ & 63764 \\
SEU & $9.5 \%$ & 42968 \\
SET & $9.89 \%$ & 57339 \\
NUM & $9.55 \%$ & 22779 \\
ALG & $11.08 \%$ & 54723 \\
\hline
\end{tabular}

It is interesting to note that on the SH- $\lambda$ problems Vampire-HOL-RCUoff-CEoff which treats combinators as uninterpreted symbols and cannot synthesise higher-order functions outperforms Leo-III suggesting that this problem set is highly first-order in nature. This may well explain why RCU performs poorly across the problem set in general (adding it as an option to the schedule actually reduces the number of proofs). We suspect that RCU is becoming trapped in the high-order portions of the problems which do not need to be explored to find a proof. The 16 problems solved by RCU that cannot be solved with combinators show that RCU is still a valuable option to include in the schedule. However, for these mostly first-order problems, the schedule requires tweaking to reduce the prominence of RCU.

Amongst the TPTP benchmarks, Vampire solves 31 problems not solved by LeoIII and Satallax $3^{3}$ Out of these, 2 (SEV016^5 5 and SEV032^5) are difficulty rating 1.00 problems, meaning that they are unsolvable by any current theorem prover. Amongst the 31 problems 17 are solved by strategies that utilise combinatory unification, showing its value. On the SH- $\lambda$ benchmarks, Vampire solves 55 problems that Leo-III and Satallax cannot. Out of these, 33 are solved by strategies that use combinatory unification.

Finally, we investigated the efficiency of combinatory unification. It would have been interesting to compare it against first-order unification, but this is not possible in Vampire as first order unification is carried out incrementally whilst RCU is carried out term-to-term. Instead, the amount of time spent on RCU in each run was recorded as well as the number of unifiers produced. The results can be found in Table 2 It can be seen that combinatory unification is not dominating the running time. Further the number of combinatory unifiers produced suggests that whatever the worst case complexity is, in practice the procedure is efficient.

\section{Conclusion and Related Work}

Pragmatic approaches to higher-order theorem proving include Otter- $\lambda$, a prover for lambda logic [2] developed by Michael Beeson. Lambda logic is a relatively weak extension of first-order logic. Also included is Cruanes' prover Zipperposition [8] which

\footnotetext{
${ }^{3}$ Some of these problems are marked as being solvable by Satallax on the TPTP website. However, the CASC-2018 version of Satallax that we used in our tests was unable to find a proof

${ }^{4}$ This problem can be solved by the new version of E, Ehoh developed by Vukmirović et al.
} 
he extended to HOL. The prover rewrites using the combinator definitions which resembles our approach, but is less goal directed.

Gilles Dowek et. al. developed the deduction modulo framework [13] [7] which can be used to turn higher-order logic into a first-order theory. It involves replacing unification with unification modulo and adding rewrite rules for literals. Their proof methods don't extend to superposition and in the absence of an efficient, complete unification algorithm modulo the combinator axioms, their work remains of academic interest.

The approach we present here resembles that taken by the Leo-III higher-order prover [29]. Leo-III implements Huet's complete algorithm for higher-order unification, but then imposes a depth bound, thereby losing completeness. It is felt that RCU is more amenable to implementation within a first-order prover than depth-bound Huet's algorithm and therefore our approach is complementary. By retaining the ordering restrictions of the superposition calculus, our approach further resembles that of Leo-III which makes use of the computational path order (CPO) further losing completeness.

Related to our work is the Matryoshka team's extension of superposition to lambdafree higher-order logic [3] and, in as yet unpublished work, to full higher-order logic [4]. Their approach aims for completeness and thus has to deal with possible infinite sets of unifiers. Due to the incompleteness of our method, there will certainly be problems provable by a complete calculus that are inaccessible to us. On the other hand, it appears likely that, at least on some problems, the overhead of dealing with infinite streams of possibly redundant unifiers will allow lightweight but incomplete solutions such as RCU to outperform complete methods. However, what promises to be an interesting empirical comparison of the two approaches is future work.

As far as restrictions to higher-order unification are concerned, our approach adds to a long list of attempts to devise useful restrictions. Foremost amongst these are pattern unification [22] and its generalisation by Libal and Miller [19]. Pattern unification has gained popularity, because it is decidable and mgus exist. Bounding the number of ' $\lambda$ 's that can appear in a unifier has been shown to make higher-order unification decidable [26]. Various other restrictions have been shown to either be decidable or undecidable in [18] and [25]. As far as we are aware, we are the first to address restrictions to combinatory higher-order unification algorithms.

On the mildly higher-order Sledgehammer benchmarks, Vampire with RCU outperformed full higher-order solvers. It remains to be investigated precisely what role combinatory unification played in this. It also remains to be seen whether this results holds across larger benchmark sets. Other lines of investigation, include implementing Dougherty's algorithm, but utilising it in a limited form. Consider the following inference which we call HYPEREQRES:

$$
\frac{t_{1} \neq t_{2} \vee t_{3} \neq t_{4} \ldots \vee t_{n-1} \neq t_{n}}{[]} \text { HYPEREQRES }
$$

where Dougherty's algorithm is used to find a unifier $\theta$ that simultaneously unifies each pair of terms. As this leads directly to a refutation, the problem of redundant unifiers is circumvented. Likewise, polymorphism can be restricted to the unification algorithm. Finally, it would be interesting to evaluate the usage of substitution trees as imperfect filters. On average what percentage of terms in the index are discarded? In practice, how often does first-order unification suffice? 
Acknowledgements Thanks to Jasmin Blanchette, Alexander Bentkamp, Simon Cruanes and Petar Vukmirović for many discussions on aspects of this research. A big thanks to the Matryoshka team as a whole for sharing their benchmarks. We would also like to thank Andrei Voronkov, Michael Rawson, Alexander Steen, the maintainers of StarExec and the anonymous paper reviewers. Special thanks to Martin Riener for proof-reading the paper. The first author thanks the family of James Elson for funding his research.

\section{References}

1. F. Baader and T. Nipkow. Term Rewriting and All That. Cambridge University Press, 1999.

2. Michael Beeson. Lambda logic. In Automated Reasoning: Second International Joint Conference, IJCAR 2004, pages 4-8. Springer.

3. Alexander Bentkamp, Jasmin Christian Blanchette, Simon Cruanes, and Uwe Waldmann. Superposition for lambda-free higher-order logic. In Didier Galmiche, Stephan Schulz, and Roberto Sebastiani, editors, Automated Reasoning, pages 28-46, Cham, 2018. Springer International Publishing.

4. Alexander Bentkamp, Jasmin Christian Blanchette, Sophie Tourret, Petar Vukmirović, and Uwe Waldmann. Superposition with lambdas. submitted for publication, 2019.

5. Ahmed Bhayat and Giles Reger. Set of support for higher-order reasoning. In 6th Workshop on Practical Aspects of Automated Reasoning (PAAR), pages 2-16, 2018.

6. Chad E. Brown. Satallax: An automatic higher-order prover. In Bernhard Gramlich, Dale Miller, and Uli Sattler, editors, Automated Reasoning, pages 111-117, Berlin, Heidelberg, 2012. Springer Berlin Heidelberg.

7. Guillaume Burel. Embedding deduction modulo into a prover. In Anuj Dawar and Helmut Veith, editors, Computer Science Logic, pages 155-169, Berlin, Heidelberg, 2010. Springer Berlin Heidelberg.

8. Simon Cruanes. Superposition with structural induction. In Clare Dixon and Marcelo Finger, editors, Frontiers of Combining Systems, pages 172-188, Cham, 2017. Springer International Publishing.

9. Łukasz Czajka and Cezary Kaliszyk. Hammer for coq: Automation for dependent type theory. Journal of Automated Reasoning, 61(1):423-453, Jun 2018.

10. Flávio LC de Moura, Mauricio Ayala-Rincón, and Fairouz Kamareddine. Higher-order unification: A structural relation between huet's method and the one based on explicit substitutions. Journal of Applied Logic, 6(1):72-108, 2008.

11. Daniel J. Dougherty. Higher-order unification via combinators. Theoretical Computer Science, 114(2):273 - 298, 1993.

12. Gilles Dowek. Higher Order Unification via Explicit Substitutions. Information and Computation, 157(1-2):183-235, 2000.

13. Gilles Dowek, Thérèse Hardin, and Claude Kirchner. Theorem proving modulo. Journal of Automated Reasoning, 31(1):33-72.

14. Peter Graf. Substitution tree indexing, pages 117-131. Springer Berlin Heidelberg, Berlin, Heidelberg, 1995.

15. Kryštof Hoder and Andrei Voronkov. Comparing unification algorithms in first-order theorem proving. In Annual Conference on Artificial Intelligence, pages 435-443. Springer, 2009.

16. Gérard Huet. A unification algorithm for typed $\lambda$-calculus. Theoretical Computer Science TCS, 1:27-57, 061975. 
17. Laura Kovács and Andrei Voronkov. First-order theorem proving and Vampire. In International Conference on Computer Aided Verification, pages 1-35. Springer, 2013.

18. Jordi Levy. Decidable and undecidable second-order unification problems. In International Conference on Rewriting Techniques and Applications, pages 47-60. Springer, 1998.

19. Tomer Libal and Dale Miller. Functions-as-constructors higher-order unification. In 1st International Conference on Formal Structures for Computation and Deduction (FSCD 2016). Schloss Dagstuhl-Leibniz-Zentrum fuer Informatik, 2016.

20. Tomer Libal and Alexander Steen. Towards a Substitution Tree Based Index for Higherorder Resolution Theorem Provers. In 5th Workshop on Practical Aspects of Automated Reasoning, July 2016.

21. Jia Meng and Lawrence C. Paulson. Translating higher-order clauses to first-order clauses. Journal of Automated Reasoning, 40(1):35-60, Jan 2008.

22. Dale Miller. Unification of simply typed lambda-terms as logic programming. 1991.

23. Lawrence C Paulson and Jasmin Christian Blanchette. Three years of experience with sledgehammer, a practical link between automatic and interactive theorem provers. IWIL-2010, 1, 2010.

24. Brigitte Pientka. Higher-order term indexing using substitution trees. ACM Trans. Comput. Logic, 11(1):6:1-6:40, November 2009.

25. Christian Prehofer. Decidable higher-order unification problems. In International Conference on Automated Deduction, pages 635-649. Springer, 1994.

26. Manfred Schmidt-Schauß and Klaus U Schulz. Decidability of bounded higher-order unification. Journal of Symbolic Computation, 40(2):905-954, 2005.

27. Wayne Snyder and Jean Gallier. Higher-order unification revisited: Complete sets of transformations. Journal of Symbolic Computation, 8(1-2):101-140, 1989.

28. Alexander Steen. Extensional Paramodulation for Higher-Order Logic and its Effective Implementation Leo-III. PhD thesis, Freie Universität Berlin, 2018.

29. Alexander Steen and Christoph Benzmüller. The higher-order prover Leo-III. In International Joint Conference on Automated Reasoning, pages 108-116. Springer, 2018.

30. Aaron Stump, Geoff Sutcliffe, and Cesare Tinelli. Starexec: A cross-community infrastructure for logic solving. In Stéphane Demri, Deepak Kapur, and Christoph Weidenbach, editors, Automated Reasoning, pages 367-373, Cham, 2014. Springer International Publishing.

31. G. Sutcliffe. The TPTP problem library and associated infrastructure, from CNF to TH0, TPTP v6.4.0. Journal of Automated Reasoning, 59(4):483-502, 2017. 


\section{A Restricted Combinatory Unification is Terminating}

We prove the termination of an algorithm identical to RCU, except that reductions can be performed at all positions. That RCU is terminating follows immediately. A straightforward corollary of this is that RCU is incomplete.

Lemma 6. For any (finite) system $\mathcal{S}$, if there exists an infinite $R C U$ computation path on $\mathcal{S}$, then there exists a system $\mathcal{S}^{\prime}$ such that $\mathcal{S} \Longrightarrow^{*} \mathcal{S}^{\prime}$ and there exists an infinite computation path on $\mathcal{S}^{\prime}$ that does not include the ELIMINATE or SPLIT transforms.

Proof. Both Eliminate and Split reduce the number of unsolved variables in $\mathcal{S}$. As $\mathcal{S}$ is finite, it can only contain a finite number of unsolved variables. A case analysis of the other rules shows that either they reduce the number of unsolved variables or leave it unchanged. Thus SPLIT and EliminaTE can only be carried out a finite number of times.

Based on Lemma 6, to prove that RCU is terminating, it suffices to prove that RCU without the eliminate and split transforms is terminating. Call the resulting set of transformation rules RCU-. Next a result analogous to 6 is proved with respect to the HEADNARROW transformation.

Definition 4 (Size). The size of of a type $\sigma$ is defined inductively as follows:

- $\sigma$ is atomic, the size $(\sigma)=0$

- $\sigma=\alpha \rightarrow \beta$, then size $(\sigma)=\operatorname{size}(\alpha)+\operatorname{size}(\beta)+1$

For a term $t$, its type is denoted by $\tau(t)$

Intuitively, size $(\sigma)$ is the number of ' $\rightarrow$ 's in $\sigma$.

Lemma 7. For any (finite) system $\mathcal{S}$, if there exists an infinite $R C U$ - computation path on $\mathcal{S}$, then there exists a system $\mathcal{S}^{\prime}$ such that $\mathcal{S} \Longrightarrow{ }^{*} \mathcal{S}^{\prime}$ and there exists an infinite computation path on $\mathcal{S}^{\prime}$ that does not include the head narrow transform.

Proof. Consider the following measure on systems:

$$
\left(\sum_{v \in \operatorname{vars}(\mathcal{S})} \operatorname{size}(\tau(v)), \# \mathcal{S}\right)
$$

Where the pairs are compared lexicographically and $\# \mathcal{S}$ denotes the number of blue variables in $\mathcal{S}$ or equivalently, the cardinality of $B$. The transformation rules of RCU-, other than head narrow, keep this measure constant or reduce it. HEADNARROW reduces the measure, so there can only be a finite number of applications of head narrow. The former claim is demonstrated for a number of HEADNARROW steps:

1. KX-narrow. For a variable $X$ to be eligible for a KX-narrow step, it must have type $\alpha \rightarrow \beta$. It is replaced by a term $\mathbf{K}_{\beta \rightarrow \alpha \rightarrow \beta} X_{\beta}^{\prime}$. Clearly size $\left(\tau\left(X^{\prime}\right)\right)<\operatorname{size}(\tau(X))$ and so the first item of the measure is reduced.

2. BX-narrow. For a variable $X$ to be eligible for a BX-narrow step, it must have type $(\alpha \rightarrow \beta) \rightarrow \alpha \rightarrow \gamma$. It is replaced by a term $\mathbf{B}_{(\beta \rightarrow \gamma) \rightarrow(\alpha \rightarrow \beta) \rightarrow \alpha \rightarrow \gamma} X_{\beta \rightarrow \gamma}^{\prime}$. Again $\operatorname{size}\left(\tau\left(X^{\prime}\right)\right)<\operatorname{size}(\tau(X))$ and so the first item of the measure is reduced. 
3. CX-narrow. In this case, the size of the type of the variable being narrowed is not reduced. For a variable $X$ to be eligible for a CX-narrow step, it must have type $\alpha \rightarrow \beta \rightarrow \gamma$. It is replaced by a term $\mathbf{C}_{(\beta \rightarrow \alpha \rightarrow \gamma) \rightarrow \alpha \rightarrow \beta \rightarrow \gamma} X_{\beta \rightarrow \alpha \rightarrow \gamma}^{\prime}$. Here $\operatorname{size}\left(\tau\left(X^{\prime}\right)\right)=\operatorname{size}(\tau(X))$. However, each CX-narrow step replaces a blue variable with a red variable and therefore the second item of the measure is reduced.

Based on Lemma 7 to prove that RCU- is terminating, it suffices to prove that RCU - without the HEADNARROw transformation is terminating. This is precisely Dougherty's VT transformations which he has proven to be terminating in [11]. Therefore we have:

Theorem 2. Every sequence of RCU transformations terminates 